\title{
A Non-linear Approach to Signal Processing by Means of Vector Measure Orthogonal Functions
}

\author{
by \\ L. M. García-Raffi, E. Jiménez Fernández and E. A. Sánchez Pérez
}

\begin{abstract}
Sequences of real functions that are orthogonal with respect to a vector measure are a natural generalization of orthogonal systems with respect to a parametric measure. In this paper we develop a new procedure to construct non-linear approximations of functions by defining orthogonal series in spaces of square integrable functions with respect to a vector measure whose Fourier coefficients are also functions. We study the convergence properties of such series, defining a convenient approximation procedure for signal processing involving time dependence of the measure. Some examples involving classical orthogonal polynomials are given.
\end{abstract}

2010 Mathematics Subject Classification: Primary 46G10; Secondary 33C47, 41A46.

Keywords: signal processing, vector measures, integration.

\section{$\S 1$. Introduction}

Let $(\Omega, \Sigma)$ be a measurable space and $X$ a Banach space. Given a vector measure $\lambda: \Sigma \rightarrow X$, consider a sequence $\left(f_{n}\right)$ of (non-zero) real functions that are $\lambda$ square integrable. We say that it is orthogonal with respect to $\lambda$ if for any distinct $j, k \in \mathbb{N}, \int f_{j} f_{k} d \lambda=0$. This notion generalizes the usual orthogonality given by the integral with respect to a scalar measure, and provides a natural setting for studying the properties of functions that are orthogonal with respect to a family of measures. The analysis of such sequences has a long history, for instance

Communicated by H. Okamoto. Received April 3, 2012.

L. M. García-Raffi: Instituto Universitario de Matemática Pura y Aplicada, Camino de Vera s/n, 46022 Valencia, Spain;

e-mail: Imgarcia@mat.upv.es

E. Jiménez Fernández: Instituto Universitario de Matemática Pura y Aplicada,

Camino de Vera s/n, 46022 Valencia, Spain;

e-mail: edjimfer@mat.upv.es

E. A. Sánchez Pérez : Instituto Universitario de Matemática Pura y Aplicada, Camino de Vera s/n, 46022 Valencia, Spain;

e-mail: easancpe@mat.upv.es 
regarding orthogonal polynomials. At the end of the 19th century some relevant cases of families of polynomials that are orthogonal with respect to a large set of scalar measures - indeterminate measures - were found. The first example of such an indeterminate measure was presented by Stieltjes in 1894 (see [31]). He showed that

$$
\int_{0}^{\infty} x^{n-\log x} \sin [2 \pi \log (x)] d x=0 \quad \text { for each } n=0,1, \ldots
$$

which implies that all the densities

$$
d_{\alpha}(x)=\frac{1+\alpha \sin [2 \pi \log (x)]}{x^{\log x}}, \quad \alpha \in[-1,1],
$$

on the half-line have the same moments. The polynomials that are orthogonal with respect to this class of measures are a special case of the Stieltjes-Wigert polynomials. The study of such measures was the starting point of a mathematical theory that was first developed by Riesz and Nevanlinna and is still a fruitful research area (see for instance $\S 2.7$ in [32], and [2, 18, 21]). Using the family $\left(d_{\alpha}\right)_{\alpha \in[-1,1]}$ of densities, a vector measure can be defined in an easy way (see Example 2.2). In general this construction can be done for abstract sets of measures - for instance, parametric models in statistics - and then finding sequences of functions that are orthogonal for all the elements of a family of measures is equivalent to finding sequences that are orthogonal with respect to a vector measure.

From the point of view of vector measure theory, orthogonality with respect to a vector measure has been studied in a series of papers in the last 10 years (see $[9,10,11,20,28])$. The aim of this paper is to develop an approximation procedure for sequences of functions that are orthogonal with respect to a vector measure.

Take a signal $f(t)$ belonging to a real Hilbert space $L_{2}([a, b], \mu)$ and suppose that we want to find a good approximation for it as a series defined by functions belonging to a particular family. Suppose that the properties of the measure that models the approximation depend also on $t$, i.e. there is not a fixed measure, but a parametric family of measures, $t \rightsquigarrow \mu_{t}$. Such a parametric measure can be represented by means of a vector measure and a Bochner integrable function, and the functions that can be analyzed in this setting are exactly the ones that are 2-integrable with respect to the vector measure $\lambda$.

In this paper we show that integration with respect to vector measures provides a good framework for this kind of analysis. Using orthogonality with respect to a vector measure, we define a suitable functional structure and an error, and we prove a best approximation theorem with respect to this error. Moreover, we analyze the nature of the Bochner integrable function that determines the parametric measure $\lambda_{\phi(\omega)}$ (see Definition 3.1) and we show that the function can always be pointwise approximated in the norm of the Bochner space. 
Let us now introduce the basics of Bartle-Dunford-Schwartz integration (see for instance [19, Ch. 3]). Let $(\Omega, \Sigma)$ be a measurable space. Consider a Banach space $X$ and a countably additive vector measure $\lambda: \Sigma \rightarrow X$. A measurable function $f$ is scalarly integrable if it is integrable with respect to each scalar measure

$$
\left\langle\lambda, x^{\prime}\right\rangle(A):=\left\langle\lambda(A), x^{\prime}\right\rangle, \quad A \in \Sigma,
$$

for every $x^{\prime} \in X^{\prime}$, where $X^{\prime}$ is the space of $X$ and $B_{X^{\prime}}$ is the ball of $X^{\prime}$. We say that a scalarly integrable function $f$ is integrable with respect to $\lambda$ ( $\lambda$-integrable for short) if for each $A \in \Sigma$ there is an element $\int_{A} f d \lambda \in X$ such that $\left\langle\int_{A} f d \lambda, x^{\prime}\right\rangle=$ $\int_{A} f d\left\langle\lambda, x^{\prime}\right\rangle$ for every $x^{\prime} \in X^{\prime}$. Let $\left|\left\langle\lambda, x^{\prime}\right\rangle\right|$ be the variation of the scalar measure $\left\langle\lambda, x^{\prime}\right\rangle$. The semivariation of $\lambda$ in a set $A \in \Sigma$ is given by $\|\lambda\|(A)=\sup \left\{\left|\left\langle\lambda, x^{\prime}\right\rangle\right|(A)\right.$ : $\left.x^{\prime} \in B_{X^{\prime}}\right\}$.

A natural setting for our analysis is the space $L_{2}(\lambda)$ of $\lambda$-square integrable functions, i.e., the set of (classes of) measurable functions $f$ such that $f^{2}$ is $\lambda$ integrable, where the classes of functions are defined by means of the equivalence relation $\approx$ given by $f \approx g$ if the set where they differ has null $\lambda$-semivariation. The quantity

$$
\|f\|_{L_{2}(\lambda)}:=\sup _{x^{\prime} \in B_{X^{\prime}}}\left(\int_{\Omega}|f|^{2} d\left|\left\langle\lambda, x^{\prime}\right\rangle\right|\right)^{1 / 2}
$$

is well-defined for every $f \in L_{2}(\lambda)$ and gives a norm on $L_{2}(\lambda)$, which becomes a Banach function space in the sense of $[14$, p. 28] over a Rybakov measure for $\lambda$ when the $\lambda$-a.e. order is considered (see $[6, \mathrm{Ch}$. IX] for the definition of Rybakov measure). A product of two $\lambda$-square integrable functions is always an integrable function (see $[6,7,19,27]$ for all these definitions and results). For a positive vector measure $\lambda$ the expression $\left|\|f\|_{L_{2}(\lambda)}:=\left\|\int_{\Omega}|f|^{2} d \lambda\right\|^{1 / 2}, f \in L_{2}(\lambda)\right.$, coincides with the norm. Notice that $L_{2}(\lambda)$ is not in general isomorphic to a Hilbert space, although it is always 2-convex with 2 -convexity constant 1 (see [19, Proposition $3.28]$ ).

Let $g \in L_{2}(\lambda)$. Following the research on applications of orthonormal sequences on spaces of vector measure integrable functions (see [9, 10, 11, 20, 28]), we develop a formalism to find an approximation to $g$ with respect to a distance defined using the norm $\|\cdot\|_{L_{2}(\lambda)}$ by means of products of special sequences of functions $\left(f_{i}\right)_{i=1}^{\infty} \subset L_{2}(\lambda)$ and other functions $\alpha_{i}^{\phi}, i \in \mathbb{N}$, whose definition depends on a Bochner integrable function $\phi$. Essentially the Bochner integrable function provides a parametrization of a family of measures that are defined as $\left\langle\lambda, x^{\prime}\right\rangle$ for different $x^{\prime} \in X^{\prime}$. The functions $\alpha_{i}^{\phi}$ will be called pointwise dependent Fourier coefficients, since the construction is closely related to the usual way of defining these coefficients in the Hilbert space formalism. However, the series defined in 
this way is not given by a linear combination of the elements of an orthonormal system, since in our case the coefficients of the expansion are also functions. Thus, we obtain a non-linear approximation to the function $g$.

These ideas are presented in the five sections of this paper. After this introductory section, we establishing in Section 2 some preliminary definitions and results. Section 3 is devoted to establish our functional setting and our main approximation result (Theorem 3.7), together with some examples. We also present in that section an explicit formula for the pointwise dependent Fourier coefficients for the case where the (parametric) measure is defined by a simple function. The resulting easy representation of the coefficients motivates Section 4, where continuity of the pointwise dependent coefficients with respect to the Bochner norm is analyzed. Finally, in Section 5 we give a particular example to illustrate our technique, and we present an application to approximation of signals in acoustics.

Our main references on measure spaces, scalar measures, integration with respect to scalar measures, vector measures and function spaces are [8, 6, 14, 19]. The reader can find general definitions and results on integration with respect to vector measures and the related $L_{1}(\lambda)$ spaces in $[12,13,4]$.

\section{$\S 2$. Preliminaries}

Throughout, $\lambda: \Sigma \rightarrow X$ will be a Banach space valued countably additive vector measure. Let $\mu$ be a finite control measure for $\lambda$ that satisfies $L_{1}(\lambda) \subseteq L_{1}(\mu)$. Such a measure always exists and it can be written as a Rybakov measure for $\lambda$, i.e. $\left|\left\langle\lambda, x_{0}^{\prime}\right\rangle\right|$ for a certain $x_{0}^{\prime} \in X^{\prime}$ (see [6]). Since each $x^{\prime} \in X^{\prime}$ defines a scalar measure, we can consider Bochner integrable functions $\phi \in L_{1}\left(\mu, X^{\prime}\right)$ such that $\langle\lambda, \phi(\omega)\rangle$ is a positive measure for each $\omega \in \Omega \mu$-a.e. The notion of $\lambda$-orthogonality for sequences of functions (see $[9,10,11,28,20]$ ) becomes useful to ensure the orthogonality of the sequence with respect to every scalar product defined by each positive measure $\langle\lambda, \phi(\omega)\rangle, \omega \in \Omega$. In fact, this leads to the definition of the pointwise dependent Fourier coefficients, which are functions $\alpha_{i}: \Omega \rightarrow \mathbb{R}$.

Definition 2.1. Let $(\Omega, \Sigma)$ be a measurable space and $X$ a Banach space. Given a vector measure $\lambda: \Sigma \rightarrow X$, consider a sequence $\left(f_{i}\right)_{i=1}^{\infty}$ of real functions that are $\lambda$-square integrable. We say that $\left(f_{i}\right)_{i=1}^{\infty}$ is $\lambda$-orthogonal if

$$
\int f_{i}^{2} d \lambda \neq 0 \quad \text { for all } i \in \mathbb{N}, \quad \int f_{i} f_{j} d \lambda=0, \quad i \neq j, i, j \in \mathbb{N} .
$$

Furthermore, we say that a $\lambda$-orthonormal sequence $\left(f_{n}\right)_{n}$ is $\lambda$-orthonormal if

$$
\left\|\int f_{n}^{2} d \lambda\right\|_{X}=1 \quad \text { for all } n \in \mathbb{N}
$$


Example 2.2. The first example of an indeterminate measure was presented by Stieltjes in 1894 (see [31]). Recall that a measure $\mu$ is said to be indeterminate when the solution to the Moment Problem is not unique. For each $n \in \mathbb{N}$, consider the integrals

$$
\int_{0}^{\infty} x^{n} e^{-\ln ^{2}(x)}[1+\alpha \sin (2 \pi \ln (x))] d x=\sqrt{\pi} e^{(n+1)^{2} / 4} .
$$

If we take $|\alpha|<1$ then $\mu_{\alpha}(x)=e^{-\ln ^{2}(x)}[1+\alpha \sin (2 \pi \ln (x))]>0$ is a positive function for all $x \in\left[0, \infty\left[\right.\right.$, thus $F_{\alpha}(y)=\int_{0}^{y} e^{-\ln ^{2}(x)} x^{n}[1+\alpha \sin (2 \pi \ln (x))] d x$ is a family of non-decreasing distributions with support into $[0, \infty[$ which have the same moments $S_{n}=\sqrt{\pi} e^{(n+1)^{2} / 4}$. We consider the $(n+1) \times(n+1)$ Hankel matrix

$$
\Delta_{n}=\left[\begin{array}{ccccc}
S_{0} & S_{1} & S_{2} & \ldots & S_{n} \\
S_{1} & S_{2} & & & \vdots \\
S_{2} & & & & \\
\vdots & & & & \\
S_{n} & \ldots & & & S_{2 n}
\end{array}\right]
$$

The sequence $\left(S_{n}\right)_{n=0}^{\infty}$ is positive definite if $\operatorname{det}\left(\Delta_{n}\right)>0$ for all $n \in \mathbb{N}$. Let $\mathbf{P}[x]$ be the real polynomials of degree $n \in \mathbb{N}$. We define the linear operator $\mathfrak{L}: \mathbf{P}[x] \rightarrow \mathbb{R}$ by $\mathfrak{L}\left(Q_{n}(x)\right)=\sum_{k=0}^{n} a_{k} S_{k}$ where $Q_{n}(x)=\sum_{k=0}^{n} a_{k} x^{k}$.

Note that if the sequence $\left(S_{n}\right)_{n=0}^{\infty}$ is positive definite then the polynomial

$$
P_{n}(x)=\left|\begin{array}{cccc}
S_{0} & S_{1} & \ldots & S_{n} \\
S_{1} & \ddots & & \vdots \\
\vdots & & & \\
S_{n-1} & \ldots & & S_{2 n-1} \\
1 & x & \ldots & x^{n}
\end{array}\right|
$$

satisfies

$$
\mathfrak{L}\left(x^{k} P_{n}(x)\right)= \begin{cases}0 & \text { if } k<n, \\ \operatorname{det}\left(\Delta_{n}\right) & \text { if } k=n\end{cases}
$$

from which it follows that for every polynomial $Q_{n-1}(x)$ of degree less than or equal to $n-1$ we have

$$
\mathfrak{L}\left(Q_{n-1}(x) P_{n}(x)\right)=0 .
$$



and

It is immediate that the sequence $\left(S_{n}=\sqrt{\pi} e^{(n+1)^{2} / 4}\right)_{n=0}^{\infty}$ is positive definite

$$
\int_{0}^{\infty} x^{k} P_{n}(x) e^{-\ln ^{2}(x)}[1+\alpha \sin (2 \pi \ln (x))] d x= \begin{cases}0 & \text { if } k<n, \\ \operatorname{det}\left(\Delta_{n}\right) & \text { if } k=n,\end{cases}
$$

for every $\alpha \in \mathbb{R},|\alpha|<1$.

The above construction provides a procedure for building an $\mathbf{m}$-orthogonal sequence for a suitable vector measure $\mathbf{m}$. For instance, consider the following polynomials obtained from the moment problem presented by Stieltjes:

$$
\begin{aligned}
& p_{0}(x)=S_{0}=2.27588 \\
& p_{1}(x)=-4.81803+2.27588 x \\
& p_{2}(x)=183.457-139.22 x+15.059 x^{2}, \\
& p_{4}(x)=-655344 .+611203 . x-106211 . x^{2}+3438.93 x^{3},
\end{aligned}
$$

Now we take $\Omega=(0, \infty)$ and $\Sigma$ the $\sigma$-algebra of Lebesgue subsets of $\Omega$. We can define $\nu: \Omega \rightarrow c_{0}$ by

$$
\nu(A)=\left(\int_{A} \frac{e^{-\ln ^{2}(x)}}{m}\left[1+\frac{1}{m+1} \sin (2 \pi \ln (x))\right] d x\right)_{m=1}^{\infty},
$$

where $d x$ is the Lebesgue measure and $A \in \Sigma$. Using elementary integral calculus, it is easy to prove that for every $A \in \Sigma$,

$$
\lim _{m \rightarrow \infty} \int_{A} \frac{e^{-\ln ^{2}(x)}}{m}\left[1+\frac{1}{m+1} \sin (2 \pi \ln (x))\right] d x=0 .
$$

This shows that $\nu$ is well defined and so countably additive. Moreover, it is also clear that $p_{l} \in L_{2}(\nu)$ and for all $j<l$,

$$
\int_{0}^{\infty} p_{j}(x) p_{l}(x) d \nu=0
$$

while

$$
\left\|\int p_{l}(x) p_{l}(x) d \nu\right\|_{c_{0}}=B_{l}
$$

where $B_{l}$ is a non-null constant for all $l \in \mathbb{N}$.

Example 2.3. Let us provide another example of a $\nu$-orthogonal sequence with respect to a vector measure $\nu$. Consider the family $\left\{P_{n, k}\right\}_{n, k=1}^{\infty}$ of Stieltjes-Wigert 
polynomials. For every $n \in \mathbb{N}$ and $k \in \mathbb{N}$,

$$
P_{n, k}(x)=\frac{(-1)^{n} q(k)^{n / 2+1 / 4}}{\sqrt{\prod_{j=1}^{k}\left(1-q(k)^{j}\right)}} \sum_{i=0}^{n}\left[\left(\begin{array}{c}
n \\
i
\end{array}\right)\right]_{q} q(k)^{i^{2}}(-\sqrt{q(k)} x)^{i}
$$

where $q(k)=\exp \left(-\left(2 k^{2}\right)^{-1}\right), k$ is a positive integer and we call

$$
\left[\left(\begin{array}{c}
n \\
i
\end{array}\right)\right]_{q}=\frac{\left(1-q(k)^{n}\right)\left(1-q(k)^{n-1}\right) \cdots\left(1-q(k)^{n-i+1}\right)}{(1-q(k))\left(1-q(k)^{2}\right) \cdots\left(1-q(k)^{i}\right)},
$$

a q-binomial coefficient (also called a Gaussian coefficient or Gaussian polynomial). Let us also consider the family of normalized weights

$$
\left\{w_{k}\right\}_{k=1}^{\infty}=\left\{\frac{1}{\alpha(k) \sqrt{\pi}} k x^{-k^{2} \log x}\right\}_{k=1}^{\infty}
$$

in $[0, \infty)$, where $\alpha(k)=e^{1 / 4 k^{2}}$. It is well-known that the family of polynomials above is orthogonal in the following sense: for a fixed $k \in \mathbb{N}$, the sequence $\left(P_{n, k}\right)_{n=1}^{\infty}$ is orthogonal with weight $w_{k}$, i.e.

$$
\int_{0}^{\infty} P_{n, k}(x) P_{m, k}(x) w_{k}(x) d \mu(x)=0, \quad n \neq m
$$

(see $[32,2.7])$. Consider the Lebesgue measure space $([0, \infty), \mathcal{B}, \mu)$ and the set $\Omega_{0}=\bigcup_{k=1}^{\infty}([0, \infty) \times\{k\})$. Let $\Sigma_{0}$ be the $\sigma$-algebra given by elements of the form $A=\bigcup_{k=1}^{\infty}\left(A_{k} \times\{k\}\right) \subseteq \Omega_{0}$, where $A_{k}$ is a Lebesgue measurable subset of $[0, \infty)$ for every $k$. Let us define the vector measure $\nu: \Sigma_{0} \rightarrow c_{0}$ as

$$
\nu(A):=\sum_{k=1}^{\infty}\left(\frac{1}{k} \int_{A_{k}} w_{k}(x) d \mu(x)\right) e_{k},
$$

where $A$ is an element of $\Sigma_{0}$ as above.

Suppose now that the polynomials $P_{n, k}$ are normalized in the Hilbert space $L_{2}\left([0, \infty), w_{k} d \mu\right)$ given by the weighted measure $w_{k}(x) d \mu(x)$. Define the functions $Q_{n, k}: \Omega_{0} \rightarrow[0, \infty)$ by

$$
Q_{n, k}\left(\left(x_{j}, j\right)\right):=k^{1 / 2} P_{n, k}\left(x_{j}\right) \delta_{k, j}
$$

for $\left(x_{j}, j\right) \in \Omega_{0}$. A careful consideration of the integrals with respect to $\nu$ of products of such functions shows that

$$
\int_{\Omega_{0}} Q_{n, k} Q_{m, s} d \nu=0
$$


whenever $n \neq m$ or $k \neq s$. To see this, just take into account that if $k \neq s$, then

$$
\begin{aligned}
\int_{\Omega_{0}} Q_{n, k} Q_{m, s} d \nu= & \left(\sum_{j \neq s, k}\left(\frac{s^{1 / 2} k^{1 / 2}}{j} \int_{0}^{\infty} 0 \cdot 0 w_{j}(x) d \mu(x)\right) e_{j}\right) \\
& +\left(\frac{s^{1 / 2} k^{1 / 2}}{k} \int_{0}^{\infty} P_{n, k}(x) \cdot 0 w_{k}(x) d \mu(x)\right) e_{k} \\
& +\left(\frac{s^{1 / 2} k^{1 / 2}}{s} \int_{0}^{\infty} 0 \cdot P_{m, s}(x) w_{s}(x) d \mu(x)\right) e_{s}=0
\end{aligned}
$$

and for $k=s$ and $n \neq m$,

$$
\begin{aligned}
\int_{\Omega_{0}} Q_{n, k} Q_{m, k} d \nu= & \left(\sum_{j \neq k}\left(\frac{k}{j} \int_{0}^{\infty} 0 \cdot 0 w_{j}(x) d \mu(x)\right) e_{j}\right) \\
& +\left(\int_{0}^{\infty} P_{n, k}(x) \cdot P_{m, k}(x) w_{k}(x) d \mu(x)\right) e_{k}=0 .
\end{aligned}
$$

Therefore, every subsequence of $\left(Q_{n, k}\right)_{n, k=1}^{\infty}$ defines a $\nu$-orthonormal sequence.

\section{§3. Pointwise dependent Fourier coefficients}

In this section we develop the framework and establish the main results of the paper concerning approximation of functions. Roughly speaking, we provide the mathematical tools to define parametric measures by means of Bochner integrable functions, which leads to the definition of pointwise dependent Fourier coefficients and motivates the definition of error for our technique. We also compute the coefficients when the Bochner integrable function that defines the parametric measure is a simple function. This leads in Section 4 to show how we can compute these coefficients in the general case by approximating by the ones that hold in the simple functions case. Throughout this section $(\Omega, \Sigma, \mu)$ will be a finite measure space that controls the Banach space valued measure $\lambda: \Sigma \rightarrow X$ and $L_{1}(\lambda) \subseteq L_{1}(\mu)$.

Let $1 \leq p<\infty$. Let $\phi: \Omega \rightarrow X$ be a Bochner integrable function, i.e. a strongly measurable function such that the integral $\int_{\Omega}\|\phi(\omega)\| d \mu(\omega)$ is finite. We will use such individual functions to define the class of measures that will be considered in the following sections. We will also consider the space $L_{p}(\mu, X)$ of classes of Bochner $p$-integrable functions, that is, functions $\phi \in \mathcal{M}(\Omega, X)$ such that $\int_{\Omega}\|\phi(\omega)\|_{X}^{p} d \mu(\omega)<\infty$, where $\mathcal{M}(\Omega, X)$ is the set of all strongly measurable functions from $\Omega$ to $X$. The norm of this space is given by

$$
\|\phi\|:=\left(\int_{\Omega}\|\phi(\omega)\|_{X}^{p} d \mu(\omega)\right)^{1 / p}, \quad \phi \in L_{p}(\mu, X) .
$$


Although the functions $\phi$ as above will be considered as single functions, for approximation results we will use the Bochner norm, so $\phi$ will be considered sometimes as a representative of a class of functions. For clarity, we will occasionally use $[\phi]$ to denote the class of $\phi$. The set of (classes of) simple functions is dense in $L_{p}(\mu, X)$ (see for instance Definition 11.42 in [1, Ch. 11]).

Definition 3.1. Let $X$ be a Banach space and $X^{\prime}$ its dual space. We say that a Bochner integrable function $\phi: \Omega \rightarrow X^{\prime}$ defines a parametric measure $\lambda_{\phi(\omega)}$ if the formula

$$
\lambda_{\phi(\omega)}(A):=\langle\lambda, \phi(\omega)\rangle(A), \quad A \in \Sigma,
$$

gives a positive measure that controls $\mu$ for every $\omega \in \Omega$.

Note that for $h \in L_{1}(\lambda)$, the function $\omega \mapsto \int_{\Omega} h d \lambda_{\phi(\omega)}=\left\langle\int h d \lambda, \phi(\omega)\right\rangle$ is measurable, since by hypothesis $\phi$ is strongly (and hence weak ${ }^{*}$ ) measurable and $\int h d \lambda \in X$. This implies in particular that the functions $\alpha_{i}^{\phi}$ that appear in Definition 3.2 below are measurable. For the following definition and some purposes of this section it is enough to assume that the function $\phi$ is Gel'fand integrable (see for instance $[1,11.9]$ ). However, for the approximation procedure developed in Section 4, Bochner integrability is needed.

Definition 3.2. Consider a $\lambda$-orthogonal sequence $\left(f_{i}\right)_{i=1}^{\infty}$ and a Bochner integrable function $\phi$ that defines a parametric measure $\lambda_{\phi(\omega)}$. For each $i \in \mathbb{N}$, we define the $i$-th pointwise dependent Fourier coefficient of $g \in L_{2}(\lambda)$ as the function $\alpha_{i}^{\phi}: \Omega \rightarrow \mathbb{R}$ given by

$$
\alpha_{i}^{\phi}(\omega):=\frac{\int_{\Omega} g f_{i} d \lambda_{\phi(\omega)}}{\int_{\Omega} f_{i}^{2} d \lambda_{\phi(\omega)}} .
$$

Definition 3.2 makes sense $\mu$-a.e.: in fact, if we have two different Bochner integrable functions $\phi_{1}$ and $\phi_{2}$ that belong to the same class in $L_{1}\left(\mu, X^{\prime}\right)$, the coefficients $\alpha_{i}^{\phi_{1}}(\omega)$ and $\alpha_{i}^{\phi_{2}}(\omega)$ are equal $\mu$-a.e. However, note that these functions are defined pointwise, and will be considered in this way throughout the paper.

Definition 3.3. Let $\phi: \Omega \rightarrow X^{\prime}$ be a Bochner integrable function. We say that a sequence $\left(\beta_{i}\right)_{i=1}^{\infty}$ of measurable functions $\beta_{i}: \Omega \rightarrow \mathbb{R}$ is $\phi$-compatible with the $\lambda$-orthogonal sequence $\left(f_{i}\right)_{i=1}^{\infty}$ (compatible for short if the function $\phi$ is clear from the context) if the function $\sum_{i=1}^{\infty} \beta_{i}^{2}(\omega)\left(\int_{\Omega} f_{i}^{2} d \lambda_{\phi(\omega)}\right)$ is integrable with respect to $\mu$.

Lemma 3.4. Let $\phi: \Omega \rightarrow X^{\prime}$ be a Bochner integrable function that defines a parametric measure $\lambda_{\phi(\omega)}$. Let $\left(f_{i}\right)_{i=1}^{\infty}$ be a $\lambda$-orthogonal sequence and let $g \in L_{2}(\lambda)$. 
Then the corresponding sequence $\left(\alpha_{i}^{\phi}\right)_{i=1}^{\infty}$ of pointwise dependent Fourier coefficients is $\phi$-compatible with $\left(f_{i}\right)_{i=1}^{\infty}$.

Proof. Each function $\alpha_{i}^{\phi}$ is clearly measurable, being a quotient of measurable functions. Recall that $g \in L_{2}\left(\lambda_{\phi(\omega)}\right)$ for every $\omega \in \Omega$. Clearly for every $\omega \in \Omega$, $\sum_{i=1}^{\infty} \alpha_{i}^{\phi}(\omega) f_{i}(\eta)$ is integrable with respect to the measure $\lambda_{\phi(\omega)}$ as a consequence of the definition of $\alpha_{i}^{\phi}$ and the Hilbert space structure of $L_{2}\left(\lambda_{\phi(\omega)}\right)$ at each point $\omega$. Fix $\omega \in \Omega$. Bessel's inequality gives

$$
\begin{aligned}
\sum_{i=1}^{\infty}\left|\alpha_{i}^{\phi}(\omega)\right|^{2} \int_{\Omega} f_{i}^{2} d \lambda_{\phi(\omega)} & =\lim _{N} \sum_{i=1}^{N}\left|\alpha_{i}^{\phi}(\omega)\right|^{2} \int_{\Omega} f_{i}^{2} d \lambda_{\phi(\omega)} \\
& =\lim _{N} \sum_{i=1}^{N} \frac{\left(\int_{\Omega} g f_{i} d \lambda_{\phi(\omega)}\right)^{2}}{\int_{\Omega} f_{i}^{2} d \lambda_{\phi(\omega)}} \leq\|g\|_{L_{2}\left(\lambda_{\phi(\omega)}\right)}^{2}
\end{aligned}
$$

The function $\omega \rightsquigarrow\|g\|_{L_{2}\left(\lambda_{\phi(\omega)}\right)}^{2}$ is measurable. Thus, the computation

$$
\begin{aligned}
\|\| g\left\|_{L_{2}\left(\lambda_{\phi(\omega)}\right)}^{2}\right\|_{L^{1}(\mu)} & =\int_{\Omega}\left|\int_{\Omega} g^{2}(\eta) d \lambda_{\phi(\omega)}(\eta)\right| d \mu(\omega) \\
& =\int_{\Omega}\left\langle\int_{\Omega} g^{2}(\eta) d \lambda(\eta), \phi(\omega)\right\rangle d \mu(\omega) \\
& \leq\left\|\int_{\Omega} g^{2}(\eta) d \lambda(\eta)\right\| \cdot \int_{\Omega}\|\phi(\omega)\| d \mu(\omega) \\
& =\|g\|_{L_{2}(\lambda)}^{2} \cdot\|\phi\|_{L_{1}\left(\mu, X^{\prime}\right)}<\infty
\end{aligned}
$$

finishes the proof.

Lemma 3.5. Let $\phi: \Omega \rightarrow X^{\prime}$ be a Bochner integrable function that defines a parametric measure $\lambda_{\phi}(\omega)$. Let $\left(\beta_{i}\right)_{i=1}^{\infty}$ be a sequence of functions that is $\phi$-compatible with the $\lambda$-orthogonal sequence $\left(f_{i}\right)_{i=1}^{\infty}$ and let $g \in L_{2}(\lambda)$. Then the function

$$
\psi(\omega):=\int_{\Omega}\left(g(\eta)-\sum_{i=1}^{\infty} \beta_{i}(\omega) f_{i}(\eta)\right)^{2} d \lambda_{\phi(\omega)}(\eta)
$$

is defined $\mu$-a.e. and integrable with respect to $\mu$.

Proof. For every $\omega \in \Omega$, the definition of compatibility implies that the function $\sum_{i=1}^{\infty} \beta_{i}(\omega) f_{i}(\eta)$ is the limit of the partial sums $\sum_{i=1}^{N} \beta_{i}(\omega) f_{i}(\eta)$ in $L_{2}\left(\lambda_{\phi(\omega)}\right)$. 
Consequently, fixing $\omega$ we have

$$
\begin{aligned}
\psi(\omega):= & \lim _{N} \int_{\Omega}\left(g(\eta)-\sum_{i=1}^{N} \beta_{i}(\omega) f_{i}(\eta)\right)^{2} d \lambda_{\phi(\omega)}(\eta) \\
= & \lim _{N} \int_{\Omega}\left(g^{2}(\eta)+\sum_{i=1}^{N} \beta_{i}^{2}(\omega) f_{i}^{2}(\eta)-2 \sum_{i=1}^{N} \beta_{i}(\omega) g(\eta) f_{i}(\eta)\right) d \lambda_{\phi(\omega)}(\eta) \\
= & \int_{\Omega} g^{2} d \lambda_{\phi(\omega)}(\eta)+\sum_{i=1}^{\infty} \beta_{i}^{2}(\omega)\left(\int_{\Omega} f_{i}^{2} d \lambda_{\phi(\omega)}(\eta)\right) \\
& -2 \sum_{i=1}^{\infty} \beta_{i}(\omega) \int_{\Omega} g(\eta) f_{i}(\eta) d \lambda_{\phi(\omega)}(\eta)
\end{aligned}
$$

(for the last term of the sum just use orthogonality of $\left(f_{i}\right)_{i=1}^{N}$ and Hölder's inequality and the compatibility of $\left.\left(\beta_{i}\right)\right)$. Thus the sequence $\left(\tau_{N}\right)$ of measurable functions, where

$$
\tau_{N}(\omega):=\int_{\Omega}\left(g(\eta)-\sum_{i=1}^{N} \beta_{i}(\omega) f_{i}(\eta)\right)^{2} d \lambda_{\phi(\omega)}(\eta)
$$

converges pointwise to $\psi$ and so it is a measurable function. Moreover, the sequence $\left(\tau_{N}^{1 / 2}\right)$ is order bounded by the square integrable function

$$
h(\omega):=\left(\int_{\Omega} g^{2}(\eta) d \lambda_{\phi(\omega)}(\eta)\right)^{1 / 2}+\left(\int_{\Omega} \sum_{i=1}^{\infty} \beta_{i}^{2}(\omega) f_{i}^{2}(\eta) d \lambda_{\phi(\omega)}(\eta)\right)^{1 / 2} .
$$

The Dominated Convergence Theorem for the Lebesgue integral implies that the pointwise $\operatorname{limit} \lim _{N} \tau_{N}^{1 / 2}=\psi^{1 / 2}$ belongs to $L_{2}(\mu)$, and so $\psi \in L_{1}(\mu)$.

Lemma 3.5 allows us to give the following definition.

Definition 3.6. Let $\phi: \Omega \rightarrow X^{\prime}$ be a Bochner integrable function that defines a parametric measure $\lambda_{\phi(\omega)}$ and let $\left(f_{i}\right)_{i=1}^{\infty}$ be a $\lambda$-orthogonal sequence. Let $g \in L_{2}(\lambda)$ and consider a sequence $\left(\beta_{i}\right)_{i=1}^{\infty}$ of functions which is compatible with $\left(f_{i}\right)_{i=1}^{\infty}$. We define the error $\epsilon$ associated to the function $g \in L_{2}(\lambda)$ and the sequence $\left(\beta_{i}\right)_{i=1}^{\infty}$ by

$$
\epsilon\left(g,\left(\beta_{i}\right)_{i=1}^{\infty}\right):=\int_{\Omega}\left(\int_{\Omega}\left(g(\eta)-\sum_{i=1}^{\infty} \beta_{i}(\omega) f_{i}(\eta)\right)^{2} d \lambda_{\phi(\omega)}(\eta)\right) d \mu(\omega) .
$$

The following result is a direct consequence of the definitions and Lemma 3.5 and relates the best approximation with error $\epsilon$ to the sequence of pointwise dependent Fourier coefficients. 
Theorem 3.7. Let $\phi: \Omega \rightarrow X^{\prime}$ be a Bochner integrable function that defines a parametric measure $\lambda_{\phi(\omega)}$ and let $g \in L_{2}(\lambda)$. Let $\left(f_{i}\right)_{i=1}^{\infty}$ be a $\lambda$-orthogonal sequence. If $\left(\beta_{i}(\omega)\right)_{i=1}^{\infty}$ is a sequence of functions that is $\phi$-compatible with $\left(f_{i}\right)_{i=1}^{\infty}$, then

$$
\epsilon\left(g,\left(\alpha_{i}^{\phi}\right)_{i=1}^{\infty}\right) \leq \epsilon\left(g,\left(\beta_{i}\right)_{i=1}^{\infty}\right) .
$$

Proof. Consider the functions

$$
\psi_{\alpha}(\omega):=\left(\int_{\Omega}\left(\sum_{i=1}^{\infty} \alpha_{i}^{\phi}(\omega) f_{i}(\eta)\right)^{2} d \lambda_{\phi(\omega)}(\eta)\right)^{1 / 2}
$$

and

$$
\psi_{\beta}(\omega):=\left(\int_{\Omega}\left(\sum_{i=1}^{\infty} \beta_{i}(\omega) f_{i}(\eta)\right)^{2} d \lambda_{\phi(\omega)}(\eta)\right)^{1 / 2}
$$

and take a $\mu$-null set $A$ such that $\psi_{\alpha}$ and $\psi_{\beta}$ are defined for all $\omega \in \Omega \backslash A$. Since $g \in L_{2}(\lambda)$ we have $g \in L_{2}\left(\lambda_{\phi(\omega)}\right)$ for every $\omega \in \Omega$. The Hilbert space structure of the spaces $L_{2}\left(\lambda_{\phi(\omega)}\right)$ gives the inequalities

$$
\int_{\Omega}\left(g-\sum_{i=1}^{\infty} \alpha_{i}^{\phi}(\omega) f_{i}(\eta)\right)^{2} d \lambda_{\phi(\omega)}(\eta) \leq\left(\int_{\Omega}\left(g-\sum_{i=1}^{\infty} \beta_{i}(\omega) f_{i}(\eta)\right)^{2} d \lambda_{\phi(\omega)}(\eta)\right)
$$

for each $\omega \in \Omega$, since the pointwise dependent Fourier coefficients give the best approximation at such $\omega$ in each $L_{2}\left(\lambda_{\phi(\omega)}\right)$. Then the result is a direct consequence of the lattice properties of $L_{2}(\mu)$.

The fact that $\epsilon\left(g,\left(\alpha_{i}\right)_{i=1}^{\infty}\right)=\epsilon\left(g,\left(\beta_{i}\right)_{i=1}^{\infty}\right)$ only when $\psi_{\alpha}(\omega)=\psi_{\beta}(\omega) \mu$-a.e. is a direct consequence of the above proof.

Let $\phi_{1}$ and $\phi_{2}$ be Bochner integrable functions that define parametric measures $\lambda_{\phi_{1}}$ and $\lambda_{\phi_{2}}$. Suppose that there is a positive function $k(\omega)$ such that $\phi_{1}(\omega)=k(\omega) \phi_{2}(\omega)$. Then it is clear by the definition of the pointwise dependent Fourier coefficients that

$$
\alpha_{i}^{\phi_{1}}(\omega)=\alpha_{i}^{\phi_{2}}(\omega)
$$

for every $\omega \in \Omega$. This motivates the following definition.

Definition 3.8. We say that a strongly measurable function $\phi: \Omega \rightarrow X$ is pointwise normalized if $\|\phi(\omega)\|_{X}=1$ for every $\omega \in \Omega$. We will write $C(\mu, X)$ for the set of all such functions. Note that the set of all (classes of $\mu$-a.e. equal) functions in $C(\mu, X)$ is included in all the $L_{p}(\mu, X)$ spaces, $1 \leq p \leq \infty$.

Proposition 3.9. Let $1 \leq p<\infty$ and let $X$ be a Banach space. Then the classes of functions belonging to $C(\mu, X)$ define a closed subset in $L_{p}(\mu, X)$, and the set 
of (classes of) functions

$$
S C(\mu, X)=\left\{\sum_{i=1}^{m} v_{i} \chi_{A_{i}}:\left\|v_{i}\right\|=1,\left\{A_{i}\right\}_{i=1}^{m} \subset \Sigma \text { partition of } \Omega, m \in \mathbb{N}\right\}
$$

is dense in the set of (equivalence classes of $\mu$-a.e. equal) functions in $C(\mu, X)$, when it is considered as a subset of $L_{p}(\mu, X)$.

Proof. Consider a convergent sequence $\left(\left[\phi_{n}\right]\right)_{n=1}^{\infty}$ of classes of functions in $C(\mu, X)$ and let $\lim _{n}\left[\phi_{n}\right]=[\phi]$. Suppose that no representative $\phi$ of $[\phi]$ belongs to $C(\mu, X)$. Then for all functions in $[\phi],\|\phi(\omega)\| \neq 1$ in a measurable set $B$ such that $\mu(B) \neq 0$. Thus, there is an $\epsilon>0$ such that satisfies that

$$
\int_{B}|\|\phi\|-1|^{p} d \mu>\epsilon
$$

But this would imply, for each $n \in \mathbb{N}$,

$\int_{\Omega}\left\|\phi-\phi_{n}\right\|^{p} d \mu \geq \int_{\Omega}\left|\|\phi\|-\left\|\phi_{n}\right\|\right|^{p} d \mu=\int_{\Omega}|\|\phi\|-1|^{p} d \mu \geq \int_{B}|\|\phi\|-1|^{p} d \mu>\epsilon$,

which contradicts the fact that $\lim _{n}\left[\phi_{n}\right]=[\phi]$.

Now let us show that $S C(\mu, X)$ is dense. Let $\phi \in C(\mu, X)$. Since the set of simple functions is dense in $L_{p}(\mu, X)$, there is a sequence $\left(\phi_{n}\right)_{n=1}^{\infty}$ of simple functions such that $\lim _{n}\left[\phi_{n}\right]=[\phi]$. We can assume without loss of generality that $\left\|\phi_{n}(\omega)\right\| \neq 0$, and then we can define the sequence of pointwise normalized functions $\left(\phi_{n}(\omega) /\left\|\phi_{n}(\omega)\right\|\right)_{n=1}^{\infty}$. The inequalities

$$
\begin{aligned}
\left(\int_{\Omega}\left\|\phi-\frac{\phi_{n}}{\left\|\phi_{n}\right\|}\right\|^{p} d \mu\right)^{1 / p} & \leq\left(\int_{\Omega}\left\|\phi-\phi_{n}\right\|^{p} d \mu\right)^{1 / p}+\left(\int_{\Omega}\left|\left\|\phi_{n}\right\|-1\right|^{p} d \mu\right)^{1 / p} \\
& \leq 2\left(\int_{\Omega}\left\|\phi-\phi_{n}\right\|^{p} d \mu\right)^{1 / p}
\end{aligned}
$$

show that the sequence $\left(\left[\phi_{n}(\omega) /\left\|\phi_{n}(\omega)\right\|\right]\right)_{n=1}^{\infty}$ also converges to $[\phi]$ in $L_{p}(\mu, X)$. This finishes the proof.

We can obtain with a direct calculation an expression for the pointwise dependent Fourier coefficients when the function $\phi$ that defines the parametric measure $\lambda_{\phi(\omega)}$ is a simple function. If $\phi(\omega):=\sum_{i=1}^{m} x_{i}^{\prime} \chi_{A_{i}}(\omega)$ and $f_{j}$ is an element of the $\lambda$-orthogonal sequence $\left(f_{i}\right)_{i=1}^{\infty}$, we obtain

$$
\alpha_{j}^{\phi}(\omega)=\frac{\sum_{i=1}^{m}\left\langle\int_{\Omega} g f_{j} d \lambda, x_{i}^{\prime}\right\rangle \chi_{A_{i}}(\omega)}{\sum_{i=1}^{m}\left\langle\int_{\Omega} f_{j}^{2} d \lambda, x_{i}^{\prime}\right\rangle \chi_{A_{i}}(\omega)}=\sum_{i=1}^{m} \frac{\left\langle\int_{\Omega} g f_{j} d \lambda, x_{i}^{\prime}\right\rangle}{\left\langle\int_{\Omega} f_{j}^{2} d \lambda, x_{i}^{\prime}\right\rangle} \chi_{A_{i}}(\omega),
$$


where we have supposed that $\left\langle\int_{\Omega} f_{j}^{2} d \lambda, x_{i}^{\prime}\right\rangle \neq 0$ for every $i=1, \ldots, m$, since $\int f_{j}^{2} d \lambda_{\phi(\omega)}$ for all $\omega$ and $\left(A_{i}\right)_{i=1}^{m}$ is a disjoint sequence. In the other case, the corresponding $i$-th term does not appear in the sum.

This definition shows that the calculation of the pointwise dependent Fourier coefficients is easy when $\phi$ is a simple function. On the other hand, Proposition 3.9 implies that we can approximate every normalized function that can be used to define a parametric measure by simple functions in $S C\left(\mu, X^{\prime}\right)$. Therefore, devising an approximation procedure depends on the definition of reasonable conditions to ensure the continuity of the pointwise dependent Fourier coefficients $\alpha_{i}^{\phi}$ with respect to $\phi$ in $L_{p}\left(\mu, X^{\prime}\right)$ for a certain $1 \leq p<\infty$. We will establish such conditions in the following section.

Example 3.10. (a) Let $([-\pi, \pi], \Sigma, \mu)$ be the Lebesgue measure space and consider the vector measure $\mathbf{m}_{0}: \Sigma \rightarrow \ell_{\infty}$ given by

$$
\mathbf{m}_{0}(A):=\left(\int_{A \cap[-\pi, \pi]} \cos ^{2}(k x) d \mu\right)_{k=1}^{\infty}
$$

The measure $\mathbf{m}_{0}$ is clearly countably additive since each integral over $A$ in the definition is bounded by $\mu(A)$. Consider the functions $f_{1}(x)=\sin x, f_{2}(x)=$ $\sin 2 x, \ldots, f_{n}(x)=\sin n x, n \geq 1$. Let us show that this family of functions defines an $\mathbf{m}_{0}$-orthogonal sequence. For every couple $n, m \in \mathbb{N}$ and $k \in \mathbb{N}$ such that the expressions below are defined, we obtain the following equalities.

If $m \neq n$, then

$$
\begin{aligned}
\int f_{n} f_{m} d \mathbf{m}_{0} & =\left(\int_{-\pi}^{\pi} \sin (n x) \sin (m x) \cos ^{2}(k x) d x\right)_{k=1}^{\infty} \\
= & \left(\frac { 1 } { 8 } \left(\frac{\sin [(2 k-m-n) \pi]}{-2 k+m+n}+\frac{\sin [2(m-n) \pi]}{m-n}+\frac{\sin [(2 k+m-n) \pi]}{2 k+m-n}\right.\right. \\
& \left.\left.+\frac{\sin [(2 k-m+n) \pi]}{2 k-m+n}-\frac{2 \sin [(m+n) \pi]}{m+n}-\frac{\sin [(2 k+m+n) \pi]}{2 k+m+n}\right)\right)_{k=1}^{\infty}
\end{aligned}
$$

For $m=n$ and $k \neq n$, we get

$$
\begin{aligned}
\int f_{n} f_{n} d \mathbf{m}_{0}= & \left(\int_{-\pi}^{\pi} \sin ^{2}(n x) \cos ^{2}(k x) d x\right)_{k=1}^{\infty} \\
= & \left(\frac { 1 } { 8 } \left(4 \pi+\frac{2 \sin (2 k \pi)}{k}-\frac{\sin (2(k-n) \pi)}{k-n}\right.\right. \\
& \left.\left.-\frac{2 \sin (2 n \pi)}{n}-\frac{\sin (2(k+n)) \pi}{k+n}\right)\right)_{k=1}^{\infty}
\end{aligned}
$$

Notice that the quotients of the expressions above are 0 for some values $n, m, k$. Consider now the functions that are given for $i, j, r \in \mathbb{N}, k=2^{r}, n=3 i$ and 
$m=3 j$. Define the (countably additive) vector measure

$$
\mathbf{m}(A):=\left(\int_{A \cap[-\pi, \pi]} \frac{2}{\pi} \cos ^{2}\left(2^{r} x\right) d \mu\right)_{r=1}^{\infty} .
$$

Then, as a consequence of the calculations above,

$$
\int f_{n} f_{m} d \mathbf{m}=\left(\delta_{n m}\right)_{r=1}^{\infty}
$$

Thus, for these values, the sequence $\left(f_{3 i}\right)_{i=1}^{\infty}$ is $\mathbf{m}$-orthogonal.

(b) Let us define a parametric measure using the vector measure $\mathbf{m}$ given above. Here, $\ell_{1}$ is considered (isometrically) as a subspace of $\left(\ell_{\infty}\right)^{\prime}$. Consider the family of sets $I_{n}=\left[-\pi+\pi / 2^{n}, \pi-\pi / 2^{n}\right]$ and the standard basis $\left(e_{n}\right)$ of $\ell_{1}$. Let us take the Bochner integrable function $\phi$ given by

$$
\phi(\omega)=\sum_{n \geq 1} \chi_{I_{n} \backslash I_{n-1}}(\omega) e_{n} .
$$

It is easy to prove that this function is strongly measurable and is the limit of the sequence of simple functions $\phi_{N}(\omega):=\sum_{n=1}^{N} \chi_{I_{n} \backslash I_{n-1}}(\omega) e_{n}, N \in \mathbb{N}$. Let $g(x)=x$ and let us compute the corresponding pointwise dependent Fourier coefficients. For every $i \in \mathbb{N}$,

$$
\begin{aligned}
\alpha_{i}^{\phi}(\omega) & =\frac{\left\langle\int_{[-\pi, \pi]} g f_{i} d \mathbf{m}, \phi(\omega)\right\rangle}{\left\langle\int_{[-\pi, \pi]} f_{i}^{2} d \mathbf{m}, \phi(\omega)\right\rangle} \\
& =\sum_{r \geq 1}\left(\frac{\cos \left(\left(2^{1+r}-3 i\right) \pi\right)}{2^{1+r}-3 i}-\frac{2 \cos (3 i \pi)}{3 i}-\frac{\cos \left(\left(2^{1+r}-3 i\right) \pi\right)}{2^{1+r}+3 i}\right) \chi_{I_{r} \backslash I_{r-1}}(\omega) .
\end{aligned}
$$

Therefore, the function $h(\omega):=\sum_{i=1}^{\infty} \alpha_{i}^{\phi}(\omega) \sin (3 i \omega)$ approximates $g$.

\section{§4. Continuity of the pointwise dependent Fourier coefficients}

Take a vector measure $\lambda$, a Bocher integrable function $\phi$ and a sequence $\left(\phi_{n}\right)_{n=1}^{\infty}$ of simple functions whose classes converge to $[\phi]$ in the Bochner space $L_{1}\left(\mu, X^{\prime}\right)$. In this section we analyze the convergence properties of the pointwise dependent Fourier coefficients $\alpha_{i}^{\phi_{n}}$ to $\alpha_{i}^{\phi}$ for a given $\lambda$-orthogonal sequence $\left(f_{i}\right)_{i=1}^{\infty}$ and a function $g \in L_{2}(\lambda)$. Our aim is to show that under suitable requirements on the functions $\phi_{n}$ and $\phi$, the projections $\sum_{i=1}^{\infty} \alpha_{i}^{\phi_{n}} f_{i}$ converge to $\sum_{i=1}^{\infty} \alpha_{i}^{\phi} f_{i}$ in $L_{2}(\lambda)$.

Further assumptions are necessary to obtain the results of this section. It is important to note that our results are meaningful even in the case of finite (even short) sequences of $\lambda$-orthogonal functions, since the formulas provide a nonlinear approximation that is completely different from the usual (Hilbert space) linear approximations. In Section 5 we will show an easy example. 
Recall that we are supposing that the $\lambda$-orthogonal sequence $\left(f_{i}\right)_{i=1}^{\infty}$ belongs to $L_{2}(\mu)$ (since $L_{1}(\lambda) \subseteq L_{1}(\mu)$ ). For instance, this is just a consequence of its $\lambda$-square integrability when $\mu$ is defined as $\left\langle\lambda, x^{\prime}\right\rangle$ for a certain element $x^{\prime} \in X^{\prime}$. In general, we will assume that the vector measure $\lambda$ is equivalent to $\mu$.

We use a pointwise boundedness condition for the functions that define a parametric measure.

Definition 4.1. Let $\epsilon>0$. We say that a Bochner integrable function $\phi: \Omega \rightarrow X^{\prime}$ that defines a parametric measure $\lambda_{\phi(\omega)}$ is $\epsilon$-lower bounded with respect to the $\lambda$ orthogonal sequence $\left(f_{i}\right)_{i=1}^{\infty}$ if for every $i \in \mathbb{N}$ and $\omega \in \Omega$,

$$
\epsilon<\left\langle\int_{\Omega} f_{i}^{2} d \lambda, \phi(\omega)\right\rangle .
$$

Lemma 4.2. Let $g \in L_{2}(\lambda)$ and let $\phi \in C\left(\mu, X^{\prime}\right)$ be a Bochner integrable function that defines a parametric measure $\lambda_{\phi(\omega)}$. Assume that $\phi$ is $\epsilon$-lower bounded with respect to $\left(f_{i}\right)_{i=1}^{\infty}$ for some $\epsilon>0$. Then the Fourier coefficient $\alpha_{i}^{\phi}$ of $g$ is square integrable with respect to $\mu$ for every $i \in \mathbb{N}$. Moreover, $\left(\left[\alpha_{i}^{\phi}\right]\right)_{i=1}^{\infty}$ is a norm bounded sequence in $L_{2}(\mu)$.

Proof. Let $i \in \mathbb{N}$. The definition of the pointwise dependent Fourier coefficients gives that $\alpha_{i}^{\phi}(\omega)$ is a measurable function. Moreover,

$$
\begin{aligned}
\left(\alpha_{i}^{\phi}(\omega)\right)^{2} & =\frac{\left\langle\int_{\Omega} g f_{i} d \lambda, \phi(\omega)\right\rangle^{2}}{\left\langle\int_{\Omega} f_{i}^{2} d \lambda, \phi(\omega)\right\rangle^{2}} \leq \frac{\left\langle\int_{\Omega} g^{2} d \lambda, \phi(\omega)\right\rangle\left\langle\int_{\Omega} f_{i}^{2} d \lambda, \phi(\omega)\right\rangle}{\left\langle\int_{\Omega} f_{i}^{2} d \lambda, \phi(\omega)\right\rangle^{2}} \\
& \leq \frac{\left\|\int_{\Omega} g^{2} d \lambda\right\|\|\phi(\omega)\|}{\epsilon} .
\end{aligned}
$$

Thus,

$$
\int_{\Omega}\left(\alpha_{i}^{\phi}(\omega)\right)^{2} d \mu \leq \frac{\left\|\int_{\Omega} g^{2} d \lambda\right\|}{\epsilon}\|\phi\|_{L_{1}\left(\mu, X^{\prime}\right)} .
$$

This inequality and the well-known properties of the Banach function space $L_{2}(\mu)$ give the result.

Theorem 4.3. Let $g \in L_{2}(\lambda)$. Let $\epsilon>0$ and let $\phi \in C\left(\mu, X^{\prime}\right)$ be a function that is $\epsilon$-lower bounded with respect to the $\lambda$-orthogonal sequence $\left(f_{i}\right)_{i=1}^{\infty}$. Let $\left(\phi_{n}\right)_{n=1}^{\infty}$ be a sequence of functions in $C\left(\mu, X^{\prime}\right)$ such that $\left(\left[\phi_{n}\right]\right)_{n=1}^{\infty}$ converges to $[\phi]$ in $L_{2}\left(\mu, X^{\prime}\right)$. Then for every $i \in \mathbb{N}$,

$$
\lim _{n \rightarrow \infty}\left\|\left[\alpha_{i}^{\phi}\right]-\left[\alpha_{i}^{\phi_{n}}\right]\right\|_{L_{2}(\mu)}=0 .
$$

Proof. Let $i \in \mathbb{N}$. Then there is a natural number $n_{0}$ such that for every $n \geq n_{0}$,

$$
\epsilon<\left\langle\int_{\Omega} f_{i}^{2} d \lambda, \phi_{n}(\omega)\right\rangle
$$


$\mu$-a.e. We will simply write $\left(\phi_{n}\right)_{n=1}^{\infty}$ for the subsequence $\left(\phi_{n}\right)_{n=n_{0}}^{\infty}$. An application of Lemma 4.2 shows that $\alpha_{i}^{\phi}, \alpha_{i}^{\phi_{n}} \in L_{2}(\mu)$ for each $n \in \mathbb{N}$. Now we define

$$
h_{n}(\omega):=\frac{\left\langle\int_{\Omega} f_{i}^{2} d \lambda, \phi(\omega)\right\rangle}{\left\langle\int_{\Omega} f_{i}^{2} d \lambda, \phi_{n}(\omega)\right\rangle},
$$

for every $n \in \mathbb{N}$ and $\omega \in \Omega$ if $\left\langle\int_{\Omega} f_{i}^{2} d \lambda, \phi_{n}(\omega)\right\rangle \neq 0$, and $h_{n}(\omega)=0$ otherwise. Then the result is a direct consequence of the following calculations:

$$
\begin{aligned}
& \left\|\alpha_{i}^{\phi}-\alpha_{i}^{\phi_{n}}\right\|_{L_{2}(\mu)} \\
& \leq\left(\int_{\Omega} \frac{\left(\left\langle\int_{\Omega} g f_{i} d \lambda, \phi(\omega)\right\rangle-h_{n}(\omega)\left\langle\int_{\Omega} g f_{i} d \lambda, \phi_{n}(\omega)\right\rangle\right)^{2}}{\left\langle\int_{\Omega} f_{i}^{2} d \lambda, \phi(\omega)\right\rangle^{2}} d \mu\right)^{1 / 2} \\
& \leq\left\|\int_{\Omega} g f_{i} d \lambda\right\| \frac{1}{\epsilon}\left(\int_{\Omega}\left\|\phi-h_{n} \phi_{n}\right\|^{2} d \mu\right)^{1 / 2} \\
& \leq\left\|\int_{\Omega} g f_{i} d \lambda\right\| \frac{1}{\epsilon}\left(\int_{\Omega}\left\|\phi-\phi_{n}\right\|^{2} d \mu\right)^{1 / 2}+\left\|\int_{\Omega} g f_{i} d \lambda\right\| \frac{1}{\epsilon}\left(\int_{\Omega}\left(1-h_{n}\right)^{2}\left\|\phi_{n}\right\|^{2} d \mu\right)^{1 / 2} \\
& \leq\left\|\int_{\Omega} g f_{i} d \lambda\right\| \frac{1}{\epsilon}\left[\left(\int_{\Omega}\left\|\phi-\phi_{n}\right\|^{2} d \mu\right)^{1 / 2}+\frac{1}{\epsilon}\left\|\int_{\Omega} f_{i}^{2} d \lambda\right\|\left(\int_{\Omega}\left\|\phi-\phi_{n}\right\|^{2} d \mu\right)^{1 / 2}\right] .
\end{aligned}
$$

The results of Section 3 ensure that the set of simple functions is dense in $C\left(\mu, X^{\prime}\right)$ in all the spaces $L_{p}(\mu, X)$. Therefore, Theorem 4.3 implies that under the assumption that $\phi$ is $\epsilon$-lower bounded with respect to $\left(f_{i}\right)_{i=1}^{\infty}$, we can approximate $\mu$-a.e. each Fourier coefficient $\alpha_{i}^{\phi}(i \in \mathbb{N})$ by the sequence $\left(\alpha_{i}^{\phi_{n}}\right)_{n=1}^{\infty}$, whenever $\left(\phi_{n}\right)_{n=1}^{\infty}$ is a sequence of simple functions defining a sequence of parametric measures $\lambda_{\phi_{n}(\omega)}$ converging to $\phi$ in $L_{2}\left(\mu, X^{\prime}\right)$. If the $\lambda$-orthogonal sequence is finite, we obtain an approximation calculus under the assumptions given above.

Definition 4.4. Let $m \in \mathbb{N}$ and consider a finite $\lambda$-orthogonal sequence $\left(f_{i}\right)_{i=1}^{m}$. Let $\phi \in C\left(\mu, X^{\prime}\right)$ define a parametric measure $\lambda_{\phi(\omega)}$. Then we define the 1-error for the approximation of a function $g \in L_{2}(\lambda)$ to be

$$
E_{1}(g, \phi):=\left\|g-\sum_{i=1}^{m} \alpha_{i}^{\phi} f_{i}\right\|_{L_{1}(\mu)} \quad \mu \text {-a.e. }
$$

Corollary 4.5. Let $m \in \mathbb{N}$ and consider a function $\phi \in C\left(\mu, X^{\prime}\right)$ that defines a parametric measure $\lambda_{\phi(\omega)}$ and it is $\epsilon$-lower bounded with respect to the (finite) $\lambda$-orthogonal sequence $\left(f_{i}\right)_{i=1}^{m}$. Let $\delta>0$. Then there is a simple function $\phi^{\prime} \in$ $C\left(\mu, X^{\prime}\right)$ such that

$$
\left\|\sum_{i=1}^{m} \alpha_{i}^{\phi} f_{i}-\sum_{i=1}^{m} \alpha_{i}^{\phi^{\prime}} f_{i}\right\|_{L_{1}(\mu)} \leq \delta
$$


and consequently

$$
\left|E_{1}(g, \phi)-E_{1}\left(g, \phi^{\prime}\right)\right| \leq \delta \text {. }
$$

Proof. We just need to consider the following inequalities and apply Theorem 4.3:

$$
\begin{aligned}
\left\|\sum_{i=1}^{m} \alpha_{i}^{\phi} f_{i}-\sum_{i=1}^{m} \alpha_{i}^{\phi^{\prime}} f_{i}\right\|_{L_{1}(\mu)} & \leq \int_{\Omega}\left(\sum_{i=1}^{m}\left(\alpha_{i}^{\phi}-\alpha_{i}^{\phi^{\prime}}\right)^{2}\right)^{1 / 2}\left(\sum_{i=1}^{m} f_{i}^{2}\right)^{1 / 2} d \mu \\
& \leq\left(\int_{\Omega} \sum_{i=1}^{m}\left(\alpha_{i}^{\phi}-\alpha_{i}^{\phi^{\prime}}\right)^{2} d \mu\right)^{1 / 2}\left(\int_{\Omega} \sum_{i=1}^{m} f_{i}^{2} d \mu\right)^{1 / 2} .
\end{aligned}
$$

\section{$\S 5$. An example: approximation with finite $\lambda$-orthogonal sequences when $\lambda$ is defined by a simple function}

In this section we develop an easy numerical example in order to show the nonlinear approximation that can be obtained with our procedure. Let $\lambda: \Sigma \rightarrow X$ be a countably additive vector measure, and let $\mu$ be a finite control measure for $\lambda$ that is equivalent to it and $L_{1}(\lambda) \subseteq L_{1}(\mu)$.

Definition 5.1. We say that $\lambda$ is $\mu$-simply representable if there is a natural number $m \in \mathbb{N}$ and a simple function $\Psi(\omega):=\sum_{j=1}^{m} x_{j} \chi_{A_{j}}(\omega)$ such that $\left\{A_{j}: j=\right.$ $1, \ldots, m\}$ defines a (measurable) partition of $\Omega,\left\{x_{j}: j=1, \ldots, m\right\}$ is a linearly independent subset of $X$, and

$$
\lambda(A)=\int_{A} \Psi(\omega) d \mu=\sum_{j=1}^{m} \mu\left(A \cap A_{j}\right) x_{j}, \quad A \in \Sigma .
$$

Note that in this case the integral of a function $f \in L_{1}(\lambda)$ can be directly computed by means of the formula

$$
\int_{A} f d \lambda=\sum_{j=1}^{m}\left(\int_{A \cap A_{j}} f d \mu\right) x_{j}, \quad A \in \Sigma .
$$

If $A \in \Sigma$, we denote by $L_{2}\left(A, \Sigma_{\mid A}, \mu_{\mid A}\right)$ the function space defined by restricting the measure space $(\Omega, \Sigma, \mu)$ to $A$. In particular, we can consider functions in $L_{2}(\mu)$ as functions in $L_{2}\left(A, \Sigma_{\mid A}, \mu_{\mid A}\right)$.

Lemma 5.2. Let $\lambda$ be a $\mu$-simply representable vector measure by means of a function $\Psi:=\sum_{j=1}^{m} x_{j} \chi_{A_{j}}$ and let $\left(f_{i}\right)_{i=1}^{\infty}$ be a $\lambda$-orthogonal sequence. Then this sequence is contained in the space $L_{2}(\mu)$ and is also orthogonal in it. Moreover, it is orthogonal in each space $L_{2}\left(A_{j}, \Sigma_{\mid A_{j}}, \mu_{\mid A_{j}}\right), j=1, \ldots, m$.

Proof. A direct calculation leads to the equivalence of the norms $\|\cdot\|_{L_{2}(\mu)}$ and $\|\cdot\|_{L_{2}(\lambda)}$, and then it is easy to show that $L_{2}(\lambda)=L_{2}(\mu)$. Moreover, since the 
set $\left\{x_{j}: j=1, \ldots, m\right\}$ is linearly independent, for each $j=1, \ldots, m$ there is a functional $x_{j}^{\prime} \in X^{\prime}$ such that $\left\langle x_{j}, x_{j}^{\prime}\right\rangle=\tau_{j}>0$ and $\left\langle x_{i}, x_{j}^{\prime}\right\rangle=0$ for every $i \neq j$. Then, for every $i, j, k=1, \ldots, m$ with $i \neq j$, we obtain

$$
\tau_{k} \int_{A_{k}} f_{i} f_{j} d \mu=\left\langle\int_{\Omega} f_{i} f_{j} d \lambda, x_{k}^{\prime}\right\rangle=\left\langle 0, x_{k}^{\prime}\right\rangle=0 .
$$

This gives the result.

The following is a direct consequence of Lemma 5.2.

Lemma 5.3. Let $\lambda$ be a $\mu$-simply representable vector measure by means of a function $\Psi:=\sum_{j=1}^{m} x_{j} \chi_{A_{j}}$. Let $n \in \mathbb{N}$ and let $\left(f_{i}\right)_{i=1}^{n}$ be a $\lambda$-orthogonal sequence. Then the sequence $\left(f_{i, j}\right)_{i=1, j=1}^{n, m}$ in $L_{2}(\mu)$ given by the functions $f_{i, j}:=f_{i} \chi_{A_{j}}$, $i=1, \ldots, n, j=1, \ldots, m$, is orthogonal.

Theorem 5.4. Let $\lambda, \Psi,\left(f_{i}\right)_{i=1}^{n}$ and $\left(f_{i, j}\right)_{i=1, j=1}^{n, m}$ be as in Lemma 5.3. Then there is a Bochner integrable simple function $\Psi^{\prime}$ such that for every $g \in L_{2}(\mu)$ and every $i=1, \ldots, n$, the corresponding pointwise dependent Fourier coefficient satisfies

$$
\alpha_{i}^{\Psi^{\prime}}(\omega) f_{i}(\omega)=\sum_{j=1}^{m} \alpha_{i, j} f_{i, j}(\omega)
$$

where the constants $\alpha_{i, j}=\int_{A_{j}} g f_{i} d \mu / \int_{A_{j}} f_{i}^{2} d \mu$ are the Fourier coefficients related to the functions $f_{i, j}$.

Consequently, the projection of the function $g$ on the subspace of $L_{2}(\mu)$ generated by $\left(f_{i, j}\right)_{i=1, j=1}^{n, m}$ is given by

$$
\sum_{i=1}^{n} \alpha_{i}^{\Psi^{\prime}}(\omega) f_{i}(\omega)
$$

Proof. The function $\Psi^{\prime}$ can be defined by means of the functionals $x_{j}^{\prime}$ that appear in the proof of Lemma 5.2. Using the elements introduced there, we define

$$
\Psi^{\prime}(\omega):=\sum_{j=1}^{m} x_{j}^{\prime} \chi_{A_{j}}(\omega)
$$

Now we just compute $\alpha_{i}^{\Psi^{\prime}}(\omega)$ for every $i=1, \ldots, n$ by straightforward calculation:

$$
\alpha_{i}^{\Psi^{\prime}}(\omega)=\frac{\left\langle\int_{\Omega} g f_{i} d \lambda, \Psi^{\prime}(\omega)\right\rangle}{\left\langle\int_{\Omega} f_{i}^{2} d \lambda, \Psi^{\prime}(\omega)\right\rangle}=\frac{\sum_{j=1}^{m} \tau_{j}\left(\int_{A_{j}} g f_{i} d \mu\right) \chi_{A_{j}}}{\sum_{j=1}^{m} \tau_{j}\left(\int_{A_{j}} f_{i}^{2} d \mu\right) \chi_{A_{j}}}=\sum_{j=1}^{m}\left(\frac{\int_{A_{j}} g f_{i} d \mu}{\int_{A_{j}} f_{i}^{2} d \mu}\right) \chi_{A_{j}} .
$$

These equalities imply the result. 
We finish this section with an example and an application to approximation of signals in acoustics.

Example 5.5. Consider the 3 -dimensional Euclidean space $\ell_{3}^{2}$ and the vector measure $\lambda_{0}: \Sigma \rightarrow \ell_{3}^{2}$ given by

$$
\lambda_{0}(A):=\sum_{j=1}^{3} \mu_{0}(A \cap[j-1, j]) e_{j}, \quad A \in \Sigma,
$$

where $\left(e_{i}\right)_{i=1}^{3}$ is the basis of $\ell_{3}^{2}$ and $\left([0,3], \Sigma, \mu_{0}\right)$ is the Lebesgue measure space associated with the interval $[0,3]$. It is clear that $\lambda_{0}$ is a simply representable vector measure.

For every $x \in[0,3]$, we define the following polynomials (see Figure 1 ):

$$
\begin{aligned}
& p_{1}(x):=1, \\
& p_{2}(x):=\frac{3}{2}-\frac{11}{2} x+\frac{9}{2} x^{2}-x^{3}, \\
& p_{3}(x):=\frac{171}{175}-\frac{63}{5} x+\frac{393}{10} x^{2}-\frac{252}{5} x^{3}+\frac{309}{10} x^{4}-9 x^{5}+x^{6} .
\end{aligned}
$$

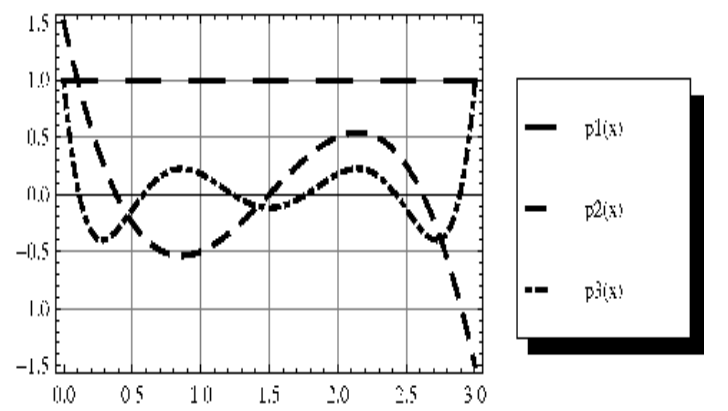

Figure 1. The functions of the $\lambda_{0}$-orthogonal sequence.

A direct calculation shows that this defines a (finite) $\lambda_{0}$-orthogonal sequence of $\lambda_{0}$-square integrable functions.

Let $g(x):=\frac{3.5}{10 x^{2}+1}+2 e^{\left(-3(x-1.5)^{2}\right)}$ be a function in $L_{2}\left(\lambda_{0}\right)$ (see Figure 2).

Let us consider different functions $\phi$ and compute the corresponding pointwise dependent Fourier coefficients $\alpha_{i}^{\phi}$. It is clear that in this case $L_{2}\left(\lambda_{0}\right)=L_{2}\left(\mu_{0}\right)$.

Let $\phi_{1}(x):=\sum_{j=1}^{3} \chi_{[j-1, j]}(x) e_{j}$. This function satisfies the conditions of Theorem 5.4 , and the approximation given by the pointwise dependent Fourier coefficients

$$
\alpha_{i}^{\phi_{1}}(x)=\sum_{j=1}^{3} \frac{\int_{[j-1, j]} p_{i} g d \mu_{0}}{\int_{[j-1, j]} p_{i}^{2} d \mu_{0}} \chi_{[j-1, j]}(x), \quad i=1,2,3,
$$




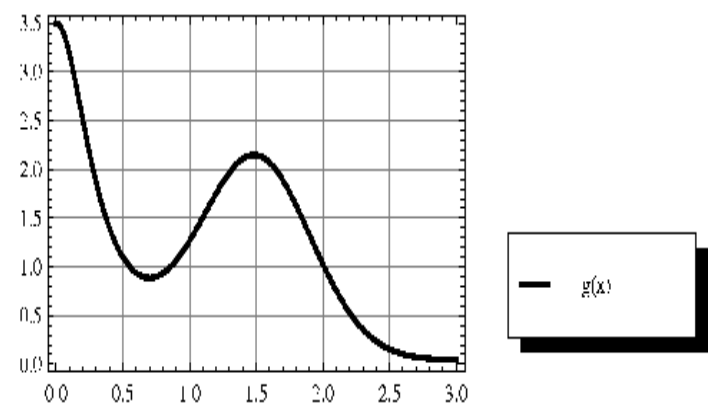

Figure 2. The function $g(x):=\frac{3.5}{10 x^{2}+1}+2 e^{\left(-3(x-1.5)^{2}\right)}$.

gives the approximation

$$
h_{1}(x):=\sum_{i=1}^{3} \alpha_{i}^{\phi_{1}}(x) p_{i}(x)
$$

to $g$ given by the projection on the subspace of $L_{2}\left(\mu_{0}\right)$ generated by the functions $p_{i, j}:=p_{i} \chi_{[j-1, j]}, i, j=1,2,3$. Note that $h_{1}$ is a linear combination of discontinuous functions and is not continuous (see Figure 3 ).

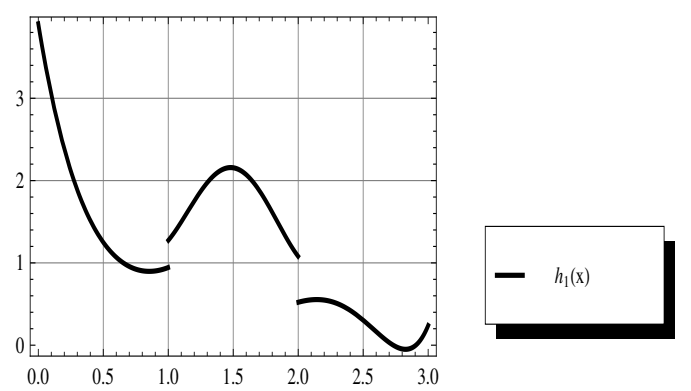

Figure 3. The function $h_{1}(x):=\sum_{j=1}^{3} \alpha_{i}^{\phi_{1}} p_{i}(x)$.

If we consider the function $\phi_{2}(x):=\chi_{[0,3]}\left(e_{1}+e_{2}+e_{3}\right) / \sqrt{3}$ and apply the same procedure (see Figure 5), we obtain a set of coefficients $\alpha_{i}^{\phi_{2}}, i=1,2,3$, that are constant functions. In fact, we obtain the coefficients of the standard approximation in the Hilbert space $L_{2}\left(\mu_{0}\right)$ by the functions $p_{1}, p_{2}$ and $p_{3}$,

$$
h_{2}(x)=\sum_{i=1}^{3} \alpha_{i}^{\phi_{2}} p_{i}(x)=1.22283 p_{1}(x)+0.735055 p_{2}(x)+0.104471 p_{3}(x) .
$$




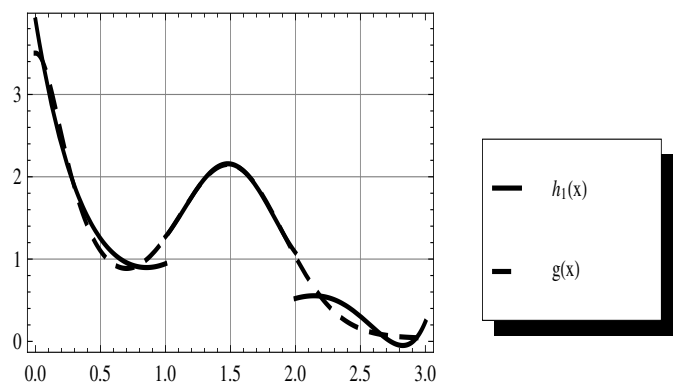

Figure 4. The function $g(x)$ and the approximation $h_{1}(x)$ using the parametrization corresponding to $\phi_{1}(x)$.

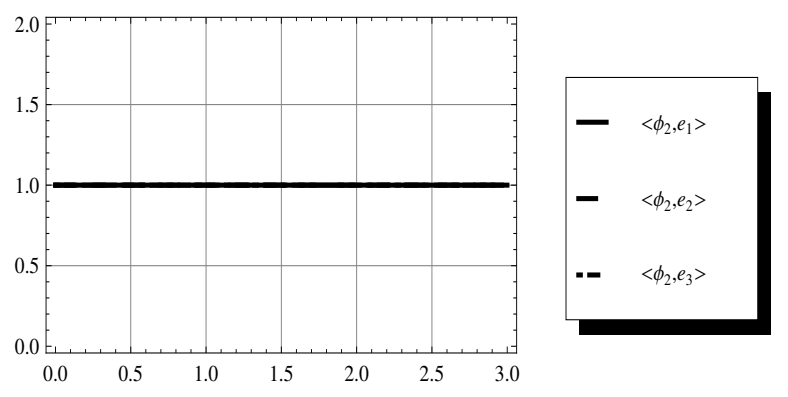

Figure 5 . The graph of the projections of the function $\phi_{2}(x)$. All of them coincide.

However $h_{2}$ does not provide a good approximation to the function $g$. We continue by testing and we can obtain a continuous function that approximates $g$. Consider the function

$$
\psi(x):=\sum_{j=1}^{3} \exp \left(-\left(\frac{x-(j-1 / 2)}{0.4}\right)^{2}\right) e_{j},
$$

and define (see Figure 7)

$$
\phi_{3}(x):=\frac{\psi(x)}{\|\psi(x)\|}, \quad x \in[0,3] .
$$

In this case, the pointwise dependent Fourier coefficients that give the approximation $h_{3}(x)=\sum_{i=1}^{3} \alpha_{i}^{\phi_{3}}(x) p_{i}(x)$ are

$$
\alpha_{i}^{\phi_{3}}(x)=\frac{\sum_{j=1}^{3}\left(\int_{[j-1, j]} f_{i} g d \mu_{0}\right) \exp \left(-\left(\frac{x-(j-1 / 2)}{0.4}\right)^{2}\right)}{\sum_{j=1}^{3}\left(\int_{[j-1, j]} f_{i}^{2} d \mu_{0}\right) \exp \left(-\left(\frac{x-(j-1 / 2)}{0.4}\right)^{2}\right)}, \quad i=1,2,3 .
$$




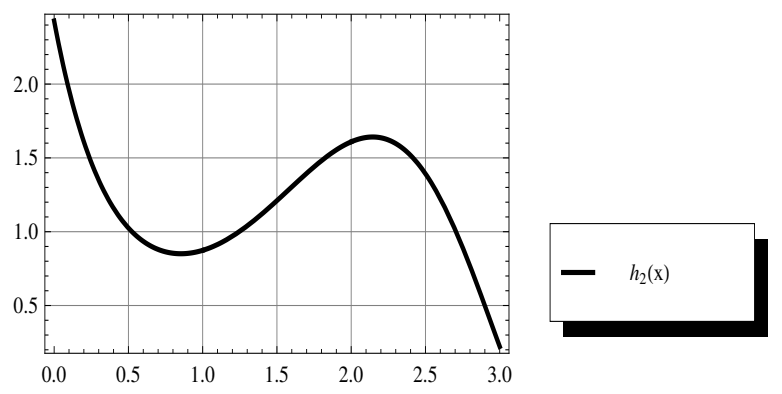

Figure 6. The function $h_{2}(x):=\sum_{j=1}^{3} \alpha_{i}^{\phi_{1}} p_{i}(x)$.

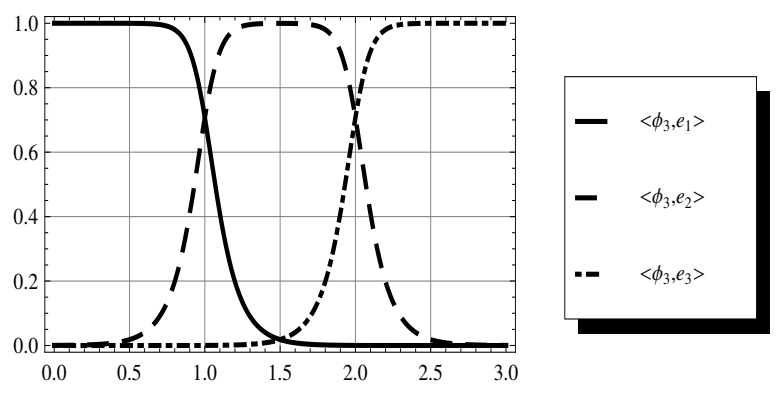

Figure 7. The graphs of the projections of $\phi_{3}(x)$.

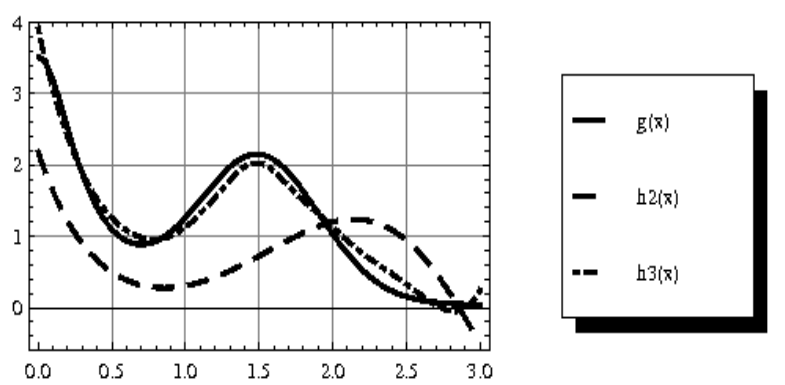

Figure 8. $g(x)$ and the approximations $h_{2}(x)$ and $h_{3}(x)$.

Figure 8 shows $g(x)$ and the approximations $h_{2}(x)$ and $h_{3}(x)$ that are continuous by the construction.

The results on the continuity of the pointwise dependent Fourier coefficients can be applied to obtain the corresponding errors of this kind of approximation. If $\phi, \phi^{\prime} \in L_{1}\left(\mu, X^{\prime}\right)$ are such that there is an $\epsilon>0$ such that both $\phi$ and $\phi^{\prime}$ are 
$\epsilon$-lower bounded with respect to the corresponding $\lambda$-orthogonal sequence (as in the case of the example), we can obtain bounds for the distances $\left\|\alpha_{i}^{\phi}-\alpha_{i}^{\phi^{\prime}}\right\|_{L_{2}(\mu)}$ in terms of $\left\|\phi-\phi^{\prime}\right\|_{L_{1}\left(\mu, X^{\prime}\right)}$ following the technique given in the proof of Theorem 4.3. Therefore, we can also obtain a bound for the $L_{1}(\mu)$-norm of the difference between the approximations associated to the corresponding functions $\phi$ and $\phi^{\prime}$, the 1-errors $E_{1}(g, \phi)$ and $E_{1}\left(g, \phi^{\prime}\right)$ and a bound for the difference between them using the procedure given in the proof of Corollary 4.5.

Remark 5.6. As a concluding remark, we point out that Figure 5 shows that our approach is fundamentally different from the classical one. This procedurethat weighs every function $f_{i}$ with a Fourier coefficient that depends on the parametrized measure and it is in fact a function itself depending also on the variable - produces a non-linear approximation of the original function $g$. That is, suppose that our function $g$ is the result of sampling a signal during a time interval of length $\Delta T$ (in the preceding example, the interval is [0,3], i.e., $\Delta T=3$ ). Then we choose functions $f_{i}$ (in the example $\left.\left\{p_{1}(x), p_{2}(x), p_{3}(x)\right\}\right)$ according to the shape of $g$ and we can produce two approximations shown in the example. Suppose that these three functions are so chosen that the Hilbert space approximation is very good. Now we sample the signal again and we obtain a new function $g$. It is not guaranteed that the same functions $f_{i}$ will be suitable for this new approximation of $g$. In fact, Hilbert approximation can be very bad. In our approach, parametrized Fourier coefficients avoid this and allow one to keep the same bases of functions during iterative approximations. Consequently, our procedure is a natural framework for a dynamic approximation of functions depending on one parameter, for example time. In the following section, we show how this technique can be applied to a real-world problem.

\section{§5.1. Application to real data}

In the previous sections we have developed our approximation considering a function $g$ that mimics signals that appear in some applied areas, like physics. In this section we are going to present an analysis of a true signal coming from the field of acoustics. In order to be consistent we are going to use the same sequence of functions as in the previous section. We will see that our technique is so flexible that functions that are not adequate "a priori" because their shape does not fit the shape of the signal, can still be used in our case. Our approximation produces better results that the corresponding standard approximation.

We are going to use a particular example related to acoustics, more precisely to sonic crystals (SC). These are periodic arrangements of scatterers embedded in a medium with different physical properties, in our case cylinders of a rigid 
material embedded in air [17]. These structures, when considered as an infinite periodic medium (an eigenvalue problem), present ranges of frequencies where the transmission of waves is forbidden. These ranges are known as band gaps (BGs). In the last years, there is an increasing interest in the potential exploitation of SC as environmental noise barriers [30], [26]. However, the acoustical properties of SC depend on several factors showing some particularities in their attenuation properties. For example, the size and position of the BGs depend on several factors such as the direction of incidence of the wave on the SC and the type of arrangement of the scatterers [29]. As a consequence, the development of screens based on SC is not a trivial process.

When a finite structure is considered, the correct understanding of these attenuation bands requires multiple scattering theory [3], [33]. Then waves are not transmitted through the structures due to the appearance of evanescent modes (see $[22,23,25])$. In this section we are dealing with one of these evanescent modes. The physical nature of such modes remains elusive and recent developments have allowed to clarify part of their nature. But the comparison of experimental measurements with theoretical models implies having good approximations of such a kind of signals.

In this section we show that orthogonal functions with respect to vector measures could be an interesting tool in studying such phenomena. If we measure the acoustic pressure between two rows of a SC, we observe a signal corresponding to an evanescent mode (see Figure 9), that is, a mode whose amplitude decreases as

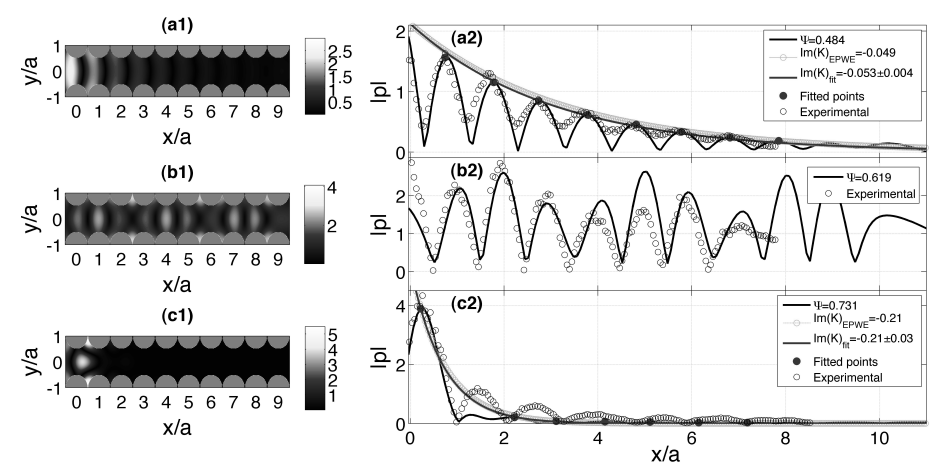

Figure 9. Evanescent modes. Both top and bottom panels show examples of evanescent modes. The exponential decay of the modes can be observed. The central panel shows a normal oscillating mode propagated through the cristal. The figure shows the experimental measurement of the acoustic field between two rows of the crystal. 
the wave passes through the periodic structure. Figure 9 shows the experimental measurement of the acoustic field between two rows of the crystal. The top and bottom panels show examples of these evanescent modes where one can see the exponential decay (fitted using the values of the pressure at the maxima) of the modes when the impinging wave propagates through the cristal. In comparison, the central panel shows a normal oscillating mode propagated through the cristal.

For comparison with the preceding approximations we use the same finite sequence of $\lambda_{0}$-orthogonal functions ( $\lambda_{0}$ defined as before; see Example 5.5), shown in Figure 1 and also the same parametrizations $\phi_{2}(x)$, that provide the same coefficients as the standard approximation in the Hilbert space $L_{2}\left(\mu_{0}\right)$ by the functions $p_{1}, p_{2}$ and $p_{3}$, and $\phi_{3}(x)$. The signal to be approximated is represented in Figure 10.

If we apply our approximation with parametrization corresponding to $\phi_{2}(x)$, we obtain the result shown in Figure 11. The result is not surprising because "a priori" the shape of the functions considered in our approximation is unsuitable for the shape of the signal. Keeping apart the oscillations of the signal. the approximation equivalent to the Hilbert approximations is not able to reproduce the decay of the original signal.

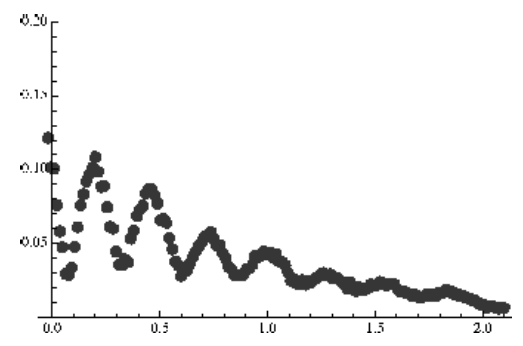

Figure 10. Data points of the signal to be approximated. These data correspond to the evanescent mode shown in the top panel of Figure 9.

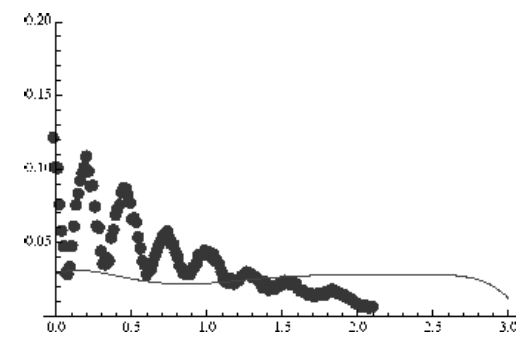

Figure 11. Hilbert-like approximation of the signal using the parametrization given by the function $\phi_{2}(x)$. 
But the parametrization using $\phi_{3}(x)$ allows us to weigh the different behaviour of the signal in the support, and the result is much more satisfactory, keeping the same finite sequence of $\lambda_{0}$-orthogonal functions $\left\{p_{1}, p_{2}, p_{3}\right\}$. The result can be seen in Figure 12.

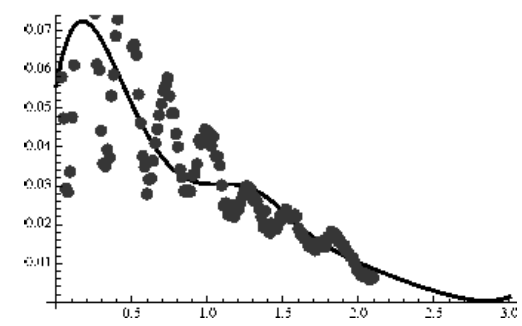

Figure 12. Vector measure non-linear approximation of the signal using the parametrization given by the function $\phi_{3}(x)$.

As a summary of this section, it is clear that a much more suitable finite sequence of functions can be found in order to have a correct approximation from the Hilbert space point of view but, when a fixed sequence has to be kept for doing the approximation of different signals whose shape can change in different time intervals, the procedure based on orthogonality with respect to a vector measure turns out to be a convenient tool that provides approximations that reproduce correctly the shape of the original signal.

\section{Acknowledgements}

L. M. García-Raffi gratefully acknowledges V. Romero-García's help with Figure 9 and the handling of experimental data.

This research was supported by Ministerio de Ciencia e Innovación, under project \#MTM2009-14483-C02-02 (Spain).

\section{References}

[1] C. D. Aliprantis and K. C. Border, Infinite dimensional analysis, Springer, Berlin, 1999. Zbl 0938.46001 MR 1717083

[2] C. Berg and G. Valent, The Nevanlinna parametrization for some indeterminate Stieltjes moment problems associated with birth and death processes, Methods Appl. Anal. 1 (1994), 169-209. Zbl 0966.44500 MR 1291292

[3] Y. Y. Chen and Z. Ye, Theoretical analysis of acoustic stop bands in two-dimensional periodic scattering arrays, Phys. Rev. E 64 (2001), 036616, 6 pp.

[4] G. P. Curbera, Banach space properties of $L_{1}$ of a vector measure, Proc. Amer. Math. Soc. 123 (1995), 3797-3806. Zbl 0848.46015 MR 1285984 
[5] A. Defant and K. Floret, Tensor norms and operator ideals, North-Holland Math. Stud. 176, North-Holland, Amsterdam, 1993. Zbl 0774.46018 MR 1209438

[6] J. Diestel and J. J. Uhl, Vector measures, Math. Surveys. 15, Amer. Math. Soc., Providence, RI, 1977. Z Zbl 0369.46039 MR 0453964

[7] A. Fernández, F. Mayoral, F. Naranjo, C. Sáez and E. A. Sánchez-Pérez, Spaces of $p$-integrable functions with respect to a vector measure, Positivity 10(2006), 1-16. Zbl 1111.46018 MR 2223581

[8] D. H. Fremlin, Measure theory, Vol. 1, Torres Fremlin, Colchester, 2000.

[9] L. M. García Raffi, D. Ginestar and E. A. Sánchez Pérez, Integration with respect to a vector measure and function approximation, Abstract Appl. Anal. 19 (2000), 207-227. Zbl 1014.46012 MR 1885467

[10] L. M. García-Raffi, D. Ginestar and E. A. Sánchez Pérez, Vector measure orthonormal systems and self-weighted functions approximation, Publ. RIMS Kyoto Univ. 41 (2005), 551-563. Zbl 1096.46025 MR 2153534

[11] L. M. García Raffi, E. A. Sánchez Pérez and J. V. Sánchez Pérez, Commutative sequences of integrable functions and best approximation with respect to the weighted vector measure distance, Integral Equations Operator Theory 54 (2006), 495-510. Zbl 1101.46028 MR 2222980

[12] D. R. Lewis, Integration with respect to vector measures, Pacific J. Math. 33 (1970), 157165. Zbl 0195.14303 MR 0259064

[13] _ On integration and summability in vector spaces, Illinois J. Math. 16 (1972), 294307. Zbl 0242.28008 MR 0291409

[14] J. Lindenstrauss and L. Tzafriri, Classical Banach spaces II, Springer, Berlin, 1979. Zbl 0403.46022 MR 0540367

[15] C. Linton and D. V. Evans, The interaction of waves with arrays of vertical circular cylinders, J. Fluid Mech. 215 (1990), 549-569. Zbl 0699.76021 MR 1061509

[16] P. A. Martin, Multiple scattering: interaction of time-harmonic waves with $N$ obstacles, Cambridge Univ. Press, 2006. Zbl 1210.35002 MR 2259988

[17] R. Martínez-Sala, J. Sancho, J. V. Sánchez-Pérez, V. Gómez, J. Llinares and F. Meseguer, Sound attenuation by sculpture, Nature 378 (1995), 241.

[18] R. Nevanlinna, Asymptotische Entwicklungen beschränkter Funktionen und das Stieltjessche Momentenproblem, Ann. Acad. Sci. Fenn. Ser. A (5) 18 (1922), no. 5, 53 pp. JFM 48.1226.02

[19] S. Okada, W. J. Ricker and E. A. Sánchez Pérez, Optimal domain and integral extension of operators acting in function spaces. Operator Theory Adv. Appl. 180, Birkhäuser, Basel, 2008. Zbl 1145.47027 MR 2418751

[20] S. Oltra, E. A. Sánchez Pérez and O. Valero, Spaces $L_{2}(\lambda)$ of a positive vector measure $\lambda$ and generalized Fourier coefficients, Rocky Mountain J. Math. 35 (2005), 211-224. Zbl 1088.46023 MR 2117604

[21] M. Riesz, Sur le problème des moments et le théorème de Parseval correspondant, Acta Sci. Math. (Szeged) 1 (1923), 209-225. JFM 49.0708.02

[22] V. Romero-García, J. V. Sánchez-Pérez, S. Castieira Ibáez and L. M. García-Raffi, Evidences of evanescent bloch waves in phononic crystals, Appl. Phys. Lett. 96 (2010), 124102.

[23] V. Romero-García, J. V. Sánchez-Pérez and L. M. García-Raffi, Modes in sonic crystals: Complex dispersion relation and supercell approximation, J. Appl. Phys. 108 (2010), 044907.

[24] , Propagating and evanescent properties of double-point defects in sonic crystals, New J. Phys. 12 (2010), 083024. 
[25] Propagating and evanescent properties of double-point defects in sonic crystals, New. J. Phys. 12 (2010), 083024.

[26] J. Sánchez-Dehesa, V. García-Chocano, D. Torrent, F. Cervera and S. Cabrera, Noise control by sonic crystal barriers made of recycled materials, arXiv:1004.2570v1 [cond-mat.mtrl-sci], 2010.

[27] E. A. Sánchez Pérez, Compactness arguments for spaces of $p$-integrable functions with respect to a vector measure and factorization of operators through Lebesgue-Bochner spaces, Illinois J. Math. 45 (2001), 907-923. Zbl 0992.46035 MR 1879243

[28] - Vector measure orthonormal functions and best approximation for the 4-norm. Arch. Math. (Basel) 80 (2003), 177-190. Zbl 1036.46031 MR 1979033

[29] J. V. Sánchez-Pérez, D. Caballero, R. Martínez-Sala, C. Rubio, J. Sánchez-Dehesa, F. Meseguer, J. Llinares and F. Gálvez, Sound attenuation by a two-dimensional array of rigid cylinders, Phys. Rev. Lett. 80 (1998), 5325.

[30] J. V. Sánchez-Pérez, C. Rubio, R. Martínez-Sala, R. Sánchez-Grandia and V. Gómez, Acoustic barriers based on periodic arrays of scatterers, Appl. Phys. Lett. 81 (2002), 5240.

[31] J. Stieltjes, Recherches sur les fractions continues, Ann. Fac. Sci. Toulouse 8 (1894), J1-J122. Zbl 0861.01036 MR 1607517

[32] G. Szegö, Orthogonal polynomials, 4th ed., Amer. Math. Soc., Providence, RI, 1975, p. 33. Zbl 0305.42011 MR 0372517

[33] F. Zaviska, Über die Beugung elektromagnetischer Wellen an parallelen, unedlich langen Kreiszylindern, Ann. Phys. 40 (1913), 1023-1056. JFM 44.102101 